\title{
Planarizing Gadgets for Perfect Matching do not Exist ${ }^{1}$
}

\author{
Rohit Gurjar $^{2} \quad$ Arpita Korwar ${ }^{2} \quad$ Jochen Messner ${ }^{3}$ \\ Simon Straub ${ }^{4} \quad$ Thomas Thierauf ${ }^{3}$
}

November 27, 2011

\begin{abstract}
To compare the complexity of the perfect matching problem for general graphs with that for planar graphs, one might try to come up with a reduction from the perfect matching problem to the planar perfect matching problem. The obvious way to construct such a reduction is via a planarizing gadget, a planar graph which replaces all edge crossings of a given graph. We show that no such gadget exists. This provides a further indication that the complexity of the two problems is different.
\end{abstract}

\section{Introduction}

The perfect matching problem is a very fundamental computational problem (see e.g. [KR98, LP86]). Edmonds [Edm65] developed a polynomial-time algorithm, but still it is unknown whether there is an efficient parallel algorithm for the perfect matching problem, i.e. whether it is in NC. In their seminal result, Mulmuley, Vazirani and Vazirani [MVV87] isolated a perfect matching by assigning random weights to the edges. This yields a randomized parallel algorithm for the problem, it is in RNC. A derandomization of this algorithm is a challenging open problem.

\footnotetext{
${ }^{1}$ Work partially supported by the Indo-German DST-DFG program, DFG grant TH 472/4-1 and DST grant DST/CS/20100251.

${ }^{2}$ IIT Kanpur, India

${ }^{3}$ Aalen University, Germany

${ }^{4}$ University of Ulm, Germany
} 
There are NC-algorithms for the perfect matching problem for special graph classes. For example for regular bipartite graphs [LPV81], dense graphs [DHK93], and strongly chordal graphs [DK86].

Here we consider planar graphs. Planarity is an intriguing property with respect to the perfect matching problems which seems to change the complexity of the problem drastically:

- Valiant [Val79] showed that counting the number of perfect matchings in a graph is a hard problem, namely it is \#P-complete,

- whereas for planar graphs, Kastelyn [Kas67] showed that a Pfaffian orientation can be computed in polynomial time, which leads to polynomial-time algorithm for counting the number of perfect matchings. Vazirani [Vaz89] argued that the problem is in fact in NC.

In contrast, no NC-algorithm is known for the construction of a perfect matching in planar graphs. This is a puzzling state of affairs because intuitively counting seems to be a harder problem than construction. There is however an RNC-algorithm for the construction problem [MVV87].

Much work has been done on the perfect matching for bipartite planar graphs [MN95, MV00, KMV08, DKR10, Hoa10, DKT11]. The current best bound on the problem is unambiguous logspace, UL, for decision and construction [DKT11]. Note that bipartiteness on its own doesn't seem to make the perfect matching problem easier: counting the number of perfect matchings in bipartite graphs is still \#P-complete. But the combination bipartite + planar seems to make a difference.

In this paper, we investigate the question whether there is logspaceor NC-reduction from the perfect matching problem to the planar perfect matching problem. Despite the differences of the problems mentioned above, such a reduction is quite possible to exist.

- Such a reduction would be a break-through result because it would derandomize the RNC-algorithm for perfect matching. Many people conjecture that such a derandomization is possible. Hence this could be one way of doing it.

- A reduction does not necessarily maintain the number of perfect matchings. Hence it does not imply an unexpected collapse of complexity classes.

An obvious approach to construct such a reduction is to come up with a planarizing gadget, a planar graph that locally replaces the crossing edges 
of a given drawing of a graph. Any other, more globally acting reduction should be very involved to construct. A recent example for the use of planarizing gadget is provided by Datta et al. [DKLM10]. They investigated the complexity of computing the determinant of a matrix which is the adjacency matrix of a planar graph. Datta et al. construct a gadget which provides a reduction from the general determinant to the planar determinant. Therefore both problems have the same complexity, they are GapL-complete. The analogous result is shown for the permanent, again via some gadget. Therefore the permanent and the planar permanent are \#P-complete.

Our main result is to put an obstacle on the way to get an $\mathrm{NC}$-algorithm for the perfect matching problem: we show that planarizing gadgets for perfect matching do not exist. We extend the result to the weighted perfect matching problem and the exact perfect matching problem.

\section{Preliminaries}

Let $G=(V, E)$ be an undirected graph. A matching in $G$ is a set $M \subseteq E$, such that no two edges in $M$ have a vertex in common. A matching $M$ is called perfect if every vertex occurs as an endpoint of some edge in $M$. In the decission problem perfect matching one has to decide, whether $G$ has a perfect matching,

$$
P M=\{G \mid G \text { has a perfect matching }\} .
$$

For a weight function $w: E \mapsto \mathbb{N}$ of the graph, the weight of a matching $M$ is defined as $w(M)=\sum_{e \in M} w(e)$.

Sequential algorithms to compute maximum matchings use augmenting path techniques [HK73]. They are described in many textbooks, see for example [Koz91, KR98]. We mention some easy facts. Let $M$ and $M^{\prime}$ be matchings in a graph $G=(V, E)$. Consider the subgraph $G^{\prime}=\left(V, M \triangle M^{\prime}\right)$ of $G$ that contains only the edges of the symmetric difference of $M$ and $M^{\prime}$. This graph consists of alternating paths and cycles (with respect to $M$ and $\left.M^{\prime}\right)$. That is, the paths and cycles have edges alternating from $M$ and $M^{\prime}$. Also, they are simple and pairwise disjoint. If $M$ and $M^{\prime}$ are perfect matchings in $G$, then $M \triangle M^{\prime}$ consists of alternating cycles only, i.e. there are no alternating paths.

We also consider the following matching problems.

- Unique perfect matching: Given a graph $G$, decide whether $G$ has precisely one perfect matching. 
- Weighted perfect matching: Given a graph $G$ and a weight function $w$, compute a perfect matching of minimum weight in $G$.

- Weighted exact perfect matching: Given a graph $G$, a weight function $w$ on the edges, and a number $W$, decide whether there is a perfect matching in $G$ of weight exactly $W$.

- Exact perfect matching: Given a graph $G$ where every edge is colored either red or blue, and a number $k$, decide whether there is a perfect matching in $G$ with exactly $k$ red edges.

The unique perfect matching problem is in $\mathrm{P}$ [GKT99]. For bipartite graphs it is in NC [KVV85, HMT06], and for planar graphs it is also in NC [Vaz89]. It is a challenging open problem whether the unique perfect matching problem is in NC.

The weighted perfect matching problem is in $\mathrm{P}$ [MV80, Vaz94]. If the weights are polynomially bounded, then the problem is in NC for planar graphs [Vaz89].

The exact perfect matching problem is a very puzzling problem: it is not even known to be in $\mathrm{P}$ (see e.g. [PY82, Yus07]). It is known to be in $\mathrm{RNC}$ [MVV87] and in $\mathrm{NC}$ for planar graphs [Vaz89]. It can be seen as special case of the weighted exact perfect matching problem: in a given redblue graph $G$, assign each red edge weight 1 and each blue edge weight 0 . Then a perfect matching with weight $k$ is a perfect matching with $k$ red edges in $G$.

The weighted exact perfect matching problem with polynomially bounded weights is (logspace-) equivalent to the exact perfect matching problem: in a given weighted graph $G$, replace each edge $e=(a, b)$ with a simple path of length $2 w(e)-1$ from $a$ to $b$. Color the edges of the path alternating red and blue with $w(e)$ red and $w(e)-1$ blue edges. Only polynomial number of edges are added. A perfect matching with $W$ red edges corresponds to a perfect matching of weight $W$ in $G$.

In contrast, the weighted exact perfect matching problem in general, i.e. with exponential weights in the number $n$ of nodes, is NP-complete [ZLM08]. In Figure 1 we present a reduction from the subset sum problem which shows that the problem becomes hard already with a trivial underlying graph structure.

The subset sum problem is known to be NP-complete even under logspace-reductions, like all the NP-complete problems in [Kar72]. Clearly, also the reduction in Figure 1 is computable in logspace, and the constructed graph is bipartite and planar. Therefore the bipartite planar weighted exact 


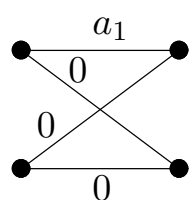

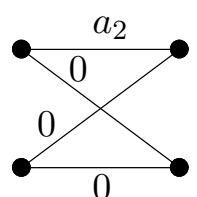

0

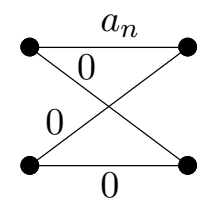

Figure 1: Reduction from subset sum to exact perfect matching. Given an instance $a_{1}, a_{2}, \ldots, a_{n}, b$ of integers for the subset sum problem, we construct the weighted graph $G$ shown above, which consists of $n$ copies of $K_{2,2}$ with weights as indicated. The subset sum instance has a solution, i.e. there is an $S \subseteq\{1, \ldots, n\}$ with $\sum_{i \in S} a_{i}=b$, if and only if $G$ has a perfect matching of weight $b$.

perfect matching problem is NP-complete under logspace reducibility. In particular, we obtain a logspace-reduction from the weighted exact perfect matching problem to its bipartite planar version. Our reduction, however, does not use any gadgets. The graph $G$ constructed consists always of a bunch of $K_{2,2}$ 's, just their number varies. Hence it is safe to say that, all the information from an instance of the general exact matching problem is encoded into the weights $a_{1}, a_{2}, \ldots, a_{n}$ of the graph $G$. This requires large weights (unless $\mathrm{P}=\mathrm{NP}$ ). With small weights only, it intuitively seems necessary that the original graph has to be encoded into the planar graph constructed in the reduction. An obvious way to do so is by an appropriate gadget!

\section{Planarizing Gadgets}

Let $G$ be a given non-planar graph and consider a drawing of $G$ in the plane. A planarizing gadget is a planar graph which is used to replace crossing edges of $G$ as shown in Figure 2. The gadget is independent of the structure of the graph. Hence, every crossing of edges is replaced by a copy of the same gadget. Let $G^{\prime}$ be the resulting planar graph.

The task of the gadget is to preserve certain properties of $G$. In our case, we want to have:

$$
G \in P M \Longleftrightarrow G^{\prime} \in P M .
$$

A gadget for which this condition holds for all graphs is called a planarizing gadget for the perfect matching problem. 

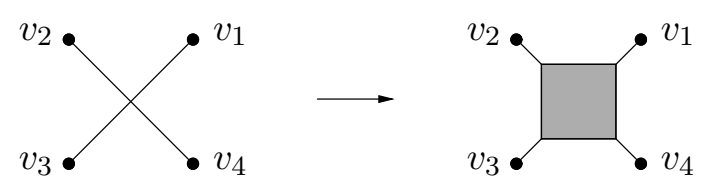

Figure 2: Planarizing gadget: the two crossing edges on the left are replaced by a planar graph which is indicated by the gray box on the right.

\subsection{Properties of the gadget}

Next, we look more closely at the properties that a planarizing gadget should fulfill. As shown in Figure 3 let $e=\left(v_{2}, v_{4}\right)$ and $e^{\prime}=\left(v_{1}, v_{3}\right)$ be crossing edges in $G$ and let $v_{i}^{\prime}$ be the node in the gadget that is connected with $v_{i}$ via edge $e_{i}$, for $i=1, \ldots, 4$.
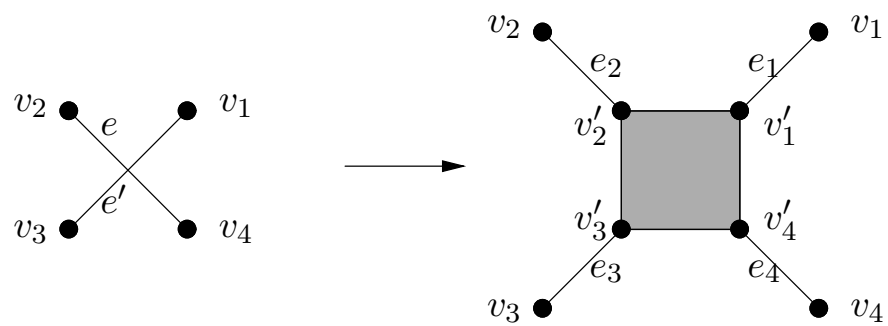

Figure 3: More details on the planarizing gadget. Nodes $v_{1}^{\prime}, \ldots, v_{4}^{\prime}$ belong to the gadget, whereas we consider edges $e_{1}, \ldots, e_{4}$ as external for the gadget.

In the positive case, when $G, G^{\prime} \in P M$, the perfect matchings of $G$ and $G^{\prime}$ should correspond to each other as follows: for a perfect matching $M$ in $G$ there is a perfect matching $M^{\prime}$ in $G^{\prime}$ such that

- $e \in M \Longleftrightarrow e_{2}, e_{4} \in M^{\prime}$ and

- $e^{\prime} \in M \Longleftrightarrow e_{1}, e_{3} \in M^{\prime}$

This enforces that if $e$ is an edge in $M$ that matches nodes $v_{2}$ and $v_{4}$, then these two nodes are matched by the gadget-replacement of $e$, namely $e_{2}, e_{4}$. Matching $M^{\prime}$ might contain further edges from inside the gadget. An analogous statement can be made for $e^{\prime}$.

Definition 1. Let $M^{\prime}$ be a matching that covers all nodes inside a gadget. Then we call $M^{\prime}$ legal for the gadget, if one of the following conditions hold: 
1. $e_{1}, e_{2}, e_{3}, e_{4} \in M^{\prime}$,

2. $e_{1}, e_{3} \in M^{\prime}$ and $e_{2}, e_{4} \notin M^{\prime}$,

3. $e_{1}, e_{3} \notin M^{\prime}$ and $e_{2}, e_{4} \in M^{\prime}$,

4. $e_{1}, e_{2}, e_{3}, e_{4} \notin M^{\prime}$.

Otherwise $M^{\prime}$ is called illegal.

Now we can characterize planarizing gadgets for the perfect matching problem.

Lemma 2. A gadget is planarizing if and only if

- there are legal matchings for all four cases described in Definition 1,

- there is no illegal matching.

Proof. Consider Figure 4. Parts (a), (b), and (c) show that the four legal

$G$<smiles>[13CH3]C([13CH3])([13CH3])[13CH3]</smiles>
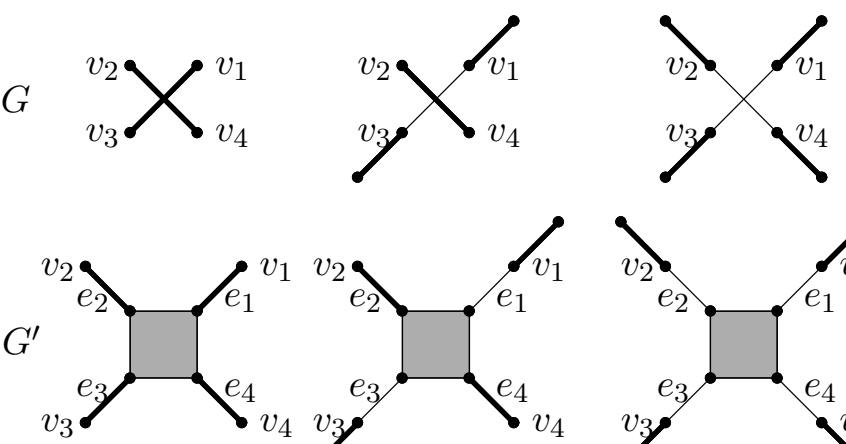

(a)

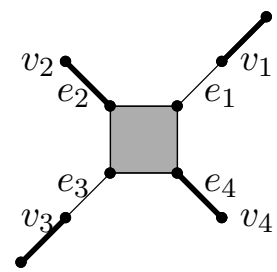

(b)

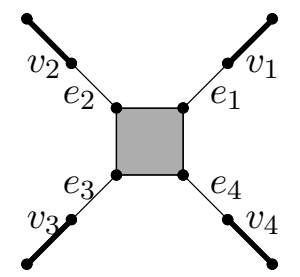

(c)
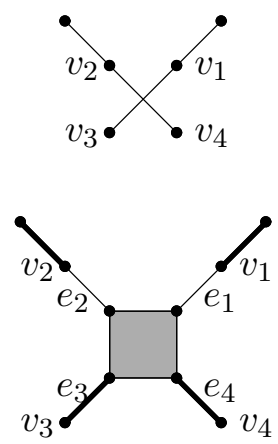

(d)

Figure 4: Graphs $G$ with a perfect matching that contains (a) both, (b) one, and (c) none of the crossing edges. Matching edges are drawn with bold lines. In $G^{\prime}$ this yields the legal matchings that contain (a) all four, (b) two opposite, and (c) none of the edges $e_{1}, \ldots, e_{4}$.

In (d), graph $G$ has no perfect matching. If the gadget would allow the illegal perfect matching that contains $e_{3}, e_{4}$ and not $e_{1}, e_{2}$, then the resulting graph $G^{\prime}$ would have a perfect matching. Hence such a gadget does not work.

cases described in Definition 1 are necessary. The case which is symmetric to part (b) is omitted. 
Note that the legal matchings imply that the gadget has an even number of nodes. Therefore we do not need to consider the case that an odd number of $e_{1}, \ldots, e_{4}$ are in the matching, Part (d) shows that a gadget which allows an illegal matching is not correct. The cases where two other neighboring edges of $e_{1}, \ldots, e_{4}$ are used are symmetric. Therefore no illegal matching is allowed to exist.

In the proof of Lemma 2, we argue with the graphs shown in Figure 4. For simplicity, these graphs are planar, but are drawn with two edges crossing. Clearly, the gadget has to work also in such cases, and hence we do not need to deal with more complicated non-planar graphs. However, it is easy to extend our graphs to non-planar graphs in such a way, that the perfect matchings are preserved: Let $G$ be one of the above graphs. For every pair of non-adjacent nodes $u, v$ in $G$, we add two additional nodes $x_{u, v}, y_{u, v}$ which are connected by an edge, and connect $u$ and $v$ with $y_{u, v}$. Let $G^{*}$ be the resulting graph. Since the only neighbor of $x_{u, v}$ is $y_{u, v}$, every perfect matching in $G^{*}$ has to use edge $\left(x_{u, v}, y_{u, v}\right)$. The other edges in the perfect matching are all from $G$. Hence perfect matchings in $G$ and $G^{*}$ differ only by the newly introduced edges $\left(x_{u, v}, y_{u, v}\right)$.

If $G$ has $n$ nodes, then $G^{*}$ has the complete graph $K_{n}$ as minor. Therefore $G^{*}$ is non-planar for $n \geq 5$. Only the graph in Figure 4 (a) has just 4 nodes. But it is easy to enlarge it by a few extra nodes and still cover the same case. Hence things do not change if we would restrict our arguments to non-planar graphs only.

Let us also note, that it suffices to consider the case where the gadget is connected to $v_{i}$ by a single edge, for $i=1, \ldots, 4$. For if there would be several connections from nodes of the gadget to $v_{i}$, we could introduce a new node $y_{i}$ to the gadget and redirect these edges to $y_{i}$ instead of $v_{i}$. Then we add one more node $x_{i}$ to the gadget and connect it via the path $\left(v_{i}, x_{i}, y_{i}\right)$. Now this modified gadget has the structure from Figure 3 and there is a direct correspondence between the matchings in both gadgets.

\subsection{Perfect Matching}

We show next that no planarizing gadget for the perfect matching problem exists. The proof constructs an illegal perfect matching out of legal ones.

Theorem 3. There is no planarizing gadget for the perfect matching problem.

Proof. Suppose there is a planarizing gadget. We refer to the denotation in Figure 3. Let $M_{1,3}, M_{2,4}$ be legal matchings such that 
1. $e_{1}, e_{3} \in M_{1,3}$ and $e_{2}, e_{4} \notin M_{1,3}$ and

2. $e_{2}, e_{4} \in M_{2,4}$ and $e_{1}, e_{3} \notin M_{2,4}$.

According to Lemma 2, a planarizing gadget should have both of these matchings.

Consider the subgraph with edges $M_{1,3} \triangle M_{2,4}$ of the gadget: as explained in the preliminary section, $M_{1,3} \triangle M_{2,4}$ consists of some alternating cycles and paths. The nodes $v_{1}, v_{2}, v_{3}, v_{4}$ must lie on alternating paths. Since the two matchings cover all nodes in the gadget, there are precisely two disjoint alternating paths $p$ and $q$, each of which connects two nodes in $\left\{v_{1}, v_{2}, v_{3}, v_{4}\right\}$. The remaining edges of $M_{1,3} \triangle M_{2,4}$ form alternating cycles.

Let us denote by $p$ the path that contains node $v_{1}$. We distinguish three cases:

(i) Suppose that $p$ connects $v_{1}$ with $v_{3}$. Therefore $q$ connects $v_{2}$ with $v_{4}$. As we assume that there is a planar drawing of the gadget where $v_{1}, v_{2}, v_{3}, v_{4}$ are placed like in Figure 3 , the two paths must cross in at least one common vertex. Since $p$ and $q$ are disjoint, this is not possible.

(ii) Suppose that $p=p_{1,2}$ connects $v_{1}$ with $v_{2}$, and $q=p_{3,4}$ connects $v_{3}$ with $v_{4}$. From $M_{1,3}$ and $M_{2,4}$ we now construct two illegal matchings $M_{2,3}$ and $M_{1,4}$ by exchanging the edges on path $p_{1,2}$ between these two sets.

Let $E\left(p_{1,2}\right)$ be the set of edges on path $p_{1,2}$. We define

$$
M_{2,3}=M_{1,3} \triangle E\left(p_{1,2}\right) .
$$

That is, in $M_{2,3}$ are

- the edges of $M_{2,4}$ that are on path $p_{1,2}$, and

- the remaining edges of $M_{1,3}$ not on path $p_{1,2}$.

Similarly we define $M_{1,4}=M_{2,4} \triangle E\left(p_{1,2}\right)$. These are the edges on path $p_{1,2}$ from $M_{1,3}$ and the other edges from $M_{1,4}$. Figure 5 gives an example of the construction.

Now both matchings $M_{1,3}$ and $M_{2,4}$ cover each inner node of the gadget, and

- $e_{2}, e_{3} \in M_{2,3}$ and $e_{1}, e_{4} \notin M_{2,3}$ and

- $e_{1}, e_{4} \in M_{1,4}$ and $e_{2}, e_{3} \notin M_{1,4}$. 


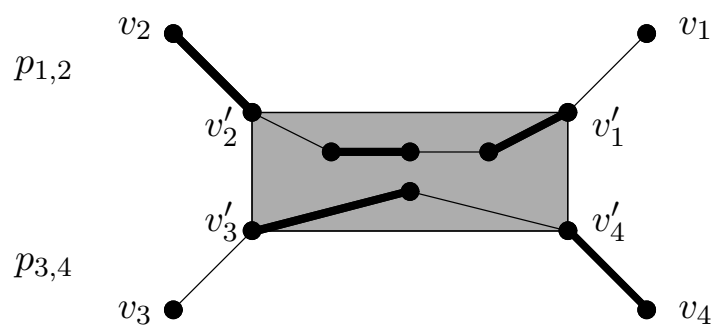

Figure 5: Matchings $M_{1,3}$ and $M_{2,4}$ are indicated, $M_{2,4}$ with bold edges. The upper alternating path $p=p_{1,2}$ connects $v_{1}$ with $v_{2}$, the lower path $q=$ $p_{3,4}$ connects $v_{3}$ with $v_{4}$. The illegal matching $M_{2,3}$ is defined as the bold edges on $p_{1,2}$ and the non-bold edges on $p_{3,4}$ and the other edges from $M_{1,3}$ that are not on these paths (not drawn here). $M_{1,4}$ consists of the remaining edges on both paths and the other edges from $M_{2,4}$.

Hence, $M_{2,3}$ and $M_{1,4}$ are illegal. Therefore, this case is not possible either.

(iii) The case that $p$ connects $v_{1}$ with $v_{4}$ is analogous to case (ii).

Hence all cases lead to a contradiction. Therefore no such gadget exists.

\subsection{Unique Perfect Matching}

We will now see whether a planarizing gadget can be constructed for the unique perfect matching problem. Such a gadget should additionally have the property that in each of the four cases of Definition 1, the matching inside the gadget must be unique. Otherwise it would not maintain uniqueness in Figure 4 (a)-(c). However, as shown in the proof of Theorem 3, we cannot avoid getting additional illegal matchings in the gadget. This destroys the uniqueness in $G^{\prime}$. Figure 6 shows a graph $G$ with a unique matching such that in the resulting graph $G^{\prime}$ an additional perfect matching can be constructed using an illegal matching in the gadget.

Corollary 4. There is no planarizing gadget for the unique perfect matching problem.

\subsection{Weighted Perfect Matching}

Next we turn to the weighted perfect matching problem. Now a planarizing gadget is allowed to have illegal matchings. But all legal matchings have to 


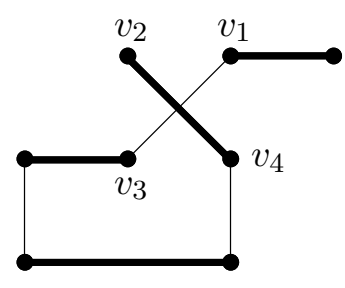

$G$

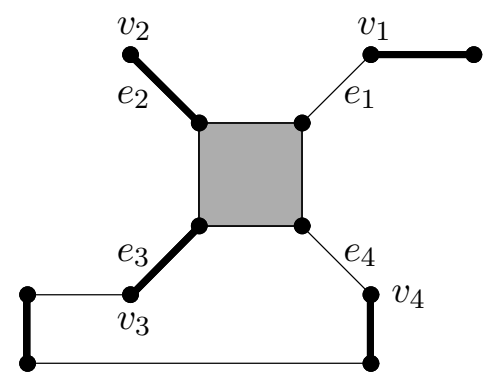

$G^{\prime}$

Figure 6: Graph $G$ has a unique perfect matching. As shown in the proof of Theorem 3, the gadget will have an illegal matching $M$ with $e_{2}, e_{3} \in M$ and $e_{1}, e_{4} \notin M$. The matching $M$ can be extended to a perfect matching in the resulting graph $G^{\prime}$. M is an additional perfect matching beside the originally unique perfect matching. The uniqueness is lost.

have weights strictly less than all illegal matchings. The proof of Theorem 3 can be extended to the weighted case. This will show that no planarizing gadget exists for the weighted perfect matching problem.

As a technical detail, we first have to define the weights of edges $e_{1}, \ldots, e_{4}$ from the weights of $e$ and $e^{\prime}$. For example we could define $w\left(e_{1}\right)=w\left(e^{\prime}\right)$, $w\left(e_{2}\right)=w(e)$, and $w\left(e_{3}\right)=w\left(e_{4}\right)=0$, or we could split the weight of $e^{\prime}$, giving part of it to $e_{1}$ and the rest to $e_{3}$, and similar for $e, e_{2}$, and $e_{4}$. For the proof below it doesn't matter which way is used.

Corollary 5. There is no planarizing gadget for the weighted perfect matching problem.

Proof. It suffices to reconsider case (ii) in the proof of Theorem 3. We have two legal matchings $M_{1,3}, M_{2,4}$ and two illegal matchings $M_{2,3}, M_{1,4}$. The illegal matchings are allowed to exist, but the weight of each illegal matching has to be strictly larger than the weight of all legal matchings.

Since $M_{2,3}$ and $M_{1,4}$ are constructed from $M_{1,3}, M_{2,4}$ solely by exchanging some edges between the two sets, i.e. $M_{2,3} \cap M_{1,4}=M_{1,3} \cap M_{2,4}$ and $M_{2,3} \triangle M_{1,4}=M_{1,3} \triangle M_{2,4}$, we have

$$
w\left(M_{2,3}\right)+w\left(M_{1,4}\right)=w\left(M_{1,3}\right)+w\left(M_{2,4}\right) .
$$

But this contradicts our assumption that the weight of $M_{2,3}, M_{1,4}$ is strictly larger than that of $M_{1,3}, M_{2,4}$. 


\subsection{Exact Perfect Matching}

Corollary 5 says that no planarizing gadget can preserve the minimum weight perfect matching. But it might still be possible that a gadget can preserve some exact weight, which is neither minimum nor maximum. That is, we ask for the existence of a gadget such that all legal matchings in the gadget have the same fixed weight, say $W_{0}$, and all illegal matchings in the gadget have weights different from $W_{0}$. Here we take only the edges inside the gadget into account, i.e. not the connecting edges $e_{1}, \ldots, e_{4}$.

If a graph $G$ is drawn with $t$ crossings, then we will have $t$ copies of the gadget in $G^{\prime}$ and the exact weight of perfect matchings increases by $t W_{0}$. However, we show that no such gadget exists as well. In the proof we construct an illegal perfect matching out of legal ones that has weight $t W_{0}$ on all the gadgets together, for $t \geq 2$. Hence such a gadget would not work correctly on all graphs.

Theorem 6. There is no planarizing gadget for the weighted exact perfect matching problem.

Proof. Let $G$ be a graph that is drawn with $t \geq 2$ crossings. Then graph $G^{\prime}$ contains $t$ gadgets. We pick two of the gadgets in $G^{\prime}$. It suffices to reconsider case (ii) in the proof of Theorem 3 for both gadgets.

- Let $M_{1,3}, M_{2,4}$ be two legal matchings and $e_{1}, \ldots, e_{4}$ be the connecting edges in the first gadget, and let $M_{2,3}, M_{1,4}$ be two illegal matchings as constructed in the proof of Theorem 3 .

- Let $M_{1,3}^{\prime}, M_{2,4}^{\prime}, M_{2,3}^{\prime}, M_{1,4}^{\prime}$ and $e_{1}^{\prime}, \ldots, e_{4}^{\prime}$ denote corresponding matchings and connecting edges in the other gadget.

Define $\stackrel{\circ}{M}_{i, j}=M_{i, j} \backslash\left\{e_{i}, e_{j}\right\}$ to be matching $M_{i, j}$ without the connecting edges $e_{i}, e_{j}$. The legal matchings all have weight $W_{0}$ inside the gadget. For example $w\left(\stackrel{\circ}{M}_{1,3}\right)=W_{0}$. The illegal matchings should have weights different from $W_{0}$ inside the gadget.

We have the following equations for the weights of the illegal matchings:

1. $w\left(\stackrel{\circ}{M}_{2,3}\right)=w\left(\stackrel{\circ}{M}_{2,3}^{\prime}\right)$ and $w\left(\stackrel{\circ}{M}_{1,4}\right)=w\left(\stackrel{\circ}{M}_{1,4}^{\prime}\right)$,

2. $w\left(\stackrel{\circ}{M}_{2,3}\right)+w\left(\stackrel{\circ}{M}_{1,4}\right)=w\left(\stackrel{\circ}{M}_{1,3}\right)+w\left(\stackrel{\circ}{M}_{2,4}\right)=2 W_{0}$.

Define matching $M=M_{2,3} \cup M_{1,4}^{\prime}$. Then $M$ covers both gadgets, is illegal, and $M$ has weight $2 W_{0}$ inside the two gadgets. Now we can extend $M$ by 
legal matchings of weight $W_{0}$ each in the other gadgets in $G^{\prime}$. This gives an illegal matching of weight $t W_{0}$ in all the gadgets together. But for the reduction to work, all legal matchings should have weight different from all illegal matchings.

In the proof of Corollary 5 and Theorem 6 we do not need to consider the weights of the graph outside the gadget. This is because we have only one gadget that should work for all graphs. But a gadget that allows for illegal matchings will be wrong on some graphs. The perfect matching problem can be embedded as a special case of the weighted and the exact perfect matching problem: define the weights of all edges of a graph with $n$ nodes to be 1 , then every perfect matching has weight $n / 2$. We have shown that there exists no planarizing gadget irrespective of the edge weights of the given graph. Thus, together with Corollary 5 and Theorem 6 , we get the following corollary.

Corollary 7. There is no planarizing gadget that reduces the perfect matching problem to the planar weighted perfect matching problem or the planar weighted exact perfect matching problem.

Similarily the exact perfect matching is a special case of the exact weighted perfect matching problem.

Corollary 8. There is no planarizing gadget for the exact perfect matching problem. Moreover, there is no planarizing gadget that reduces the exact perfect matching problem to the planar weighted exact perfect matching problem.

As a further generalization one could allow the use of several types of weighted gadgets instead of just one, and choose the gadget used at some place depending on the weights of the crossing edges it replaces. But this doesn't help: if we restrict our attention to graphs where all edges have the same weight, then we are back to the one-gadget case.

\section{Discussion}

This work shows that there is no planarizing gadget for various perfect matching problems. Although we are not able to prove the non-existence of a general (say, logspace-) reduction from arbitrary graphs to planar graphs, we prove that some plausible approach is impossible. This indicates that perfect matching might be more complex than its planar counterpart. 


\section{References}

[DHK93] E. Dahlhaus, P. Hajnal, and M. Karpinski. On the parallel complexity of hamiltonian cycles and matching problem in dense graphs. Journal of Algorithms, 15:367-384, 1993.

[DK86] E. Dahlhaus and M. Karpinski. The matching problem for strongly chordal graphs is in NC. Technical Report 855-CS, University of Bonn, 1986.

[DKLM10] S. Datta, R. Kulkarni, N. Limaye, and M. Mahajan. Planarity, determinants, permanents, and (unique) matchings. $A C M$ Trans. Comput. Theory, 1:10:1-10:20, 2010.

[DKR10] S. Datta, R. Kulkarni, and S Roy. Deterministically isolating a perfect matching in bipartite planar graphs. Theory of Computing Systems, 47:737-757, 2010.

[DKT11] S. Datta, R. Kulkarni, and R. Tewari. Perfect matching in bipartite planar graphs is in UL. Technical Report TR10-201, Electronic Colloquium on Computational Complexity, 2011.

[Edm65] J. Edmonds. Paths, trees, and flowers. Canadian Journal of Mathematics, 17:449-467, 1965.

[GKT99] H. N. Gabow, H. Kaplan, and R. E. Tarjan. Unique maximum matching algorithms. In 31st Symposium on Theory of Computing (STOC), pages 70-78. ACM Press, 1999.

[HK73] J. Hopcroft and R. Karp. An $n^{5 / 2}$ algorithm for maximum matchings in bipartite graphs. SIAM Journal on Computing, 2:225-231, 1973.

[HMT06] T.M. Hoang, M. Mahajan, and T. Thierauf. On the bipartite unique perfect matching problem. In 33rd International Colloquium on Automata, Languages and Programming (ICALP), Lecture Notes in Computer Science 4051, pages 453464. Springer-Verlag, 2006.

[Hoa10] T. M. Hoang. On the matching problem for special graph classes. In Annual IEEE Conference on Computational Complexity, pages 139-150, Los Alamitos, CA, USA, 2010. IEEE Computer Society. 
[Kar72] R. M. Karp. Reducibility among combinatorial problems. In R. E. Miller and J. W. Thatcher, editors, Complexity of Computer Computations, pages 85-103. Plenum Press, New York, 1972.

[Kas67] P. W. Kastelyn. Graph theory and crystal physics. In F. Harary, editor, Graph Theory and Theoretical Physics, pages 43-110. Academic Press, 1967.

[KMV08] R. Kulkarni, M. Mahajan, and K. Varadarajan. Some perfect matchings and perfect half-integral matchings in NC. Chicago Journal of Theoretical Computer Science, 2008(4), 2008.

[Koz91] Dexter Kozen. The Design and Analysis of Algorithms. SpringerVerlag, 1991.

[KR98] M. Karpinski and W. Rytter. Fast Parallel Algorithms for Graph Matching Problems. Oxford University Press, 1998.

[KVV85] D. C. Kozen, U. V. Vazirani, and V. V. Vazirani. NC algorithms for comparability graphs, interval graphs, and testing for unique perfect matchings. In 5th Foundations of Software Technology and Theoretical Computer Science (FSTTCS), pages 496-503. Springer-Verlag, 1985.

[LP86] L. Lovasz and M. D. Plummer. Matching theory. North-Holland, 1986 .

[LPV81] G. Lev, M. Pippenger, and L. Valiant. A fast parallel algorithm for routing in permutation networks. IEEE Transactions on Computers, 30:93-100, 1981.

[MN95] G. L. Miller and J. S. Naor. Flow in planar graphs with multiple sources and sinks. SIAM J. on Computing, 24:1002-1017, 1995.

[MV80] S. Micali and V. Vazirani. An $\mathrm{O}\left(V^{1 / 2} E\right)$ algorithm for finding maximum matching in general graphs. In IEEE Symposium on Foundations of Computer Science (FOCS), pages 17-27, 1980.

[MV00] M. Mahajan and K. R. Varadarajan. A new NC-algorithm for finding a perfect matching in bipartite planar and small genus graphs. In 32th ACM Symposium on Theory of Computing (STOC), pages 351-357. ACM Press, 2000. 
[MVV87] K. Mulmuley, U. Vazirani, and V. Vazirani. Matching is as easy as matrix inversion. Combinatorica, 7:105-113, 1987.

[PY82] C. H. Papadimitriou and M. Yannakakis. The complexity of restricted spanning tree problems. J. ACM, 29:285-309, 1982.

[Val79] L. Valiant. The complexity of computing the permanent. Theoretical Computer Science, 8:189-201, 1979.

[Vaz89] V. Vazirani. NC algorithms for computing the number of perfect matchings in $K_{3,3}$-free graphs and related problems. Information and computation, 80(2):152-164, 1989.

[Vaz94] V. Vazirani. A theory of alternating paths and blossoms for proving correctness of the $\mathrm{O}(\sqrt{(} V) E)$ general graph maximum matching algorithm. Combinatorica, 14:71-109, 1994.

[Yus07] R. Yuster. Almost exact matchings. In M. Charikar, K. Jansen, O. Reingold, and J. Rolim, editors, Approximation, Randomization, and Combinatorial Optimization. Algorithms and Techniques, volume 4627 of Lecture Notes in Computer Science, pages 286-295. Springer, 2007.

[ZLM08] G. Zhu, X. Luo, and Y. Miao. Exact weight perfect matching of bipartite graph is NP-complete. In Proceedings of the World Congress on Engineering, pages 878-880, London, U.K., 2008. 